\title{
TIME SERIES OF POLARIMETRIC AND INTERFEROMETRIC OBSERVATIONS OF TERRASAR-X DATA OVER RICE FIELDS IN SPAIN
}

\author{
Juan M. Lopez-Sanchez, J. David Ballester-Berman* \\ SST Group, DFISTS, University of Alicante
P.O. Box 99, E-03080 Alicante, Spain
E-mail: davidb@ua.es, juanma.lopez@ua.es
}

ABSTRACT

The objective of this work is to investigate the coherent copolarized behavior of rice plants during the growing stages and to explore their information content for rice monitoring at high frequencies recently available through new SAR satellite missions. Time series of dual-pol TerraSAR-X images have been acquired during the whole cultivation period over a rice site in Spain. Among different observations, the backscattering coefficients at $\mathrm{HH}$ and $\mathrm{VV}$ channels and the $\mathrm{HH} / \mathrm{VV}$ ratio have confirmed to show a temporal variation that has a significant correlation with the development of the plants during the vegetative and reproductive phenological phases. In addition, the information content of the HHVV complex coherence and a dual polarimetric target decomposition is investigated and discussed. All the information layers investigated are contributing to the discrimination of rice fields from other crop types. Finally, interferograms computed with pairs of successive images (11 days separation) have been preliminary tested.

Index Terms - Polarimetry, interferometry, agriculture.

\section{SAR DATA AND GROUND MEASUREMENTS}

The test site consists of an area of $30 \mathrm{~km} \times 30 \mathrm{~km}$ in the mouth of the Guadalquivir river, SW of Spain, where rice is cultivated annually from May to October. During the 2008 campaign, the local association of rice farmers (Federacion de Arroceros de Sevilla) has collected detailed ground measurement data on a weekly basis. For this research project, 8 specific parcels, spread over the whole site, were selected for intensive sampling. The area of each single parcel ranges from 8 to 14 ha. Phenological stage, total vegetation height, density of plants, number of tillers, and other structural parameters have been annotated for these parcels during the whole period. In addition, specific aspects for some of them have been registered, such as irrigation conditions, water salinity

*This work has been supported by the Spanish Ministry of Science and Innovation (MICINN) and EU FEDER, under Projects TEC2008-06764C02-02 and HA2007-0075, by the University of Alicante under project GRE08J01, and by Generalitat Valenciana under project GV/2009/079.

\author{
Irena Hajnsek \\ DLR, German Aerospace Center \\ P.O. Box 1116, 82234 Wessling, Germany \\ E-mail: irena.hajnsek@dlr.de
}

and presence of plagues. The final yield production of each parcel is also available. Finally, there is also climate information provided by the Spanish Government under the Sistema de Informacion Agroclimatica para el Regadio (SIAR), including daily files of temperature, precipitations, humidity and wind.

A set of three parallel time series of dual-pol stripmap images were ordered, with incidence angles of 22, 30 and 40 degrees. This specific study has been prepared by using the images with 30 degrees incidence, whose polarization channels are $\mathrm{HH}$ and VV. The dense time series provided by an 11-days revisit period enables the investigation of crop monitoring applications during the whole phenological cycle.

\section{TEMPORAL EVOLUTION OF POLSAR OBSERVABLES}

The evolutions of $\mathrm{HH}$ and VV backscattering coefficients and their ratio for all the parcels with available ground measurements are shown in Figs. 1 and 2. Since the sowing date was different for the parcels, the time series are not plotted versus the date (DoY), but shifted to a common time origin at sowing

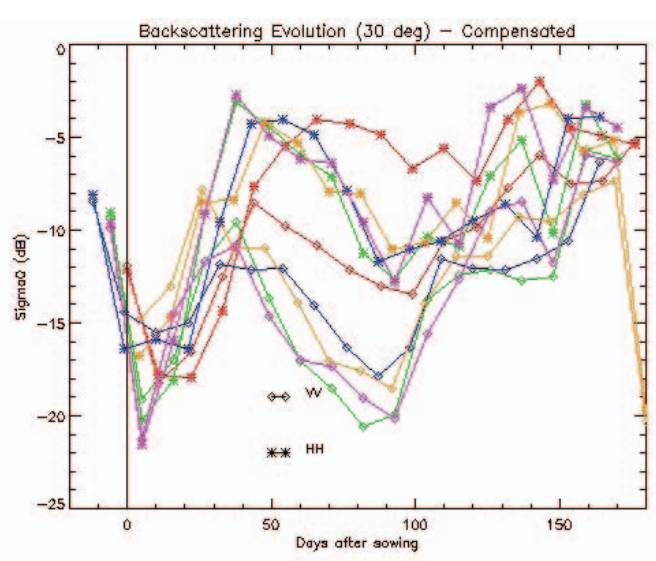

Fig. 1. Temporal evolution of the $\mathrm{HH}$ and VV backscattering coefficients for all the monitored parcels at $30^{\circ}$ incidence 


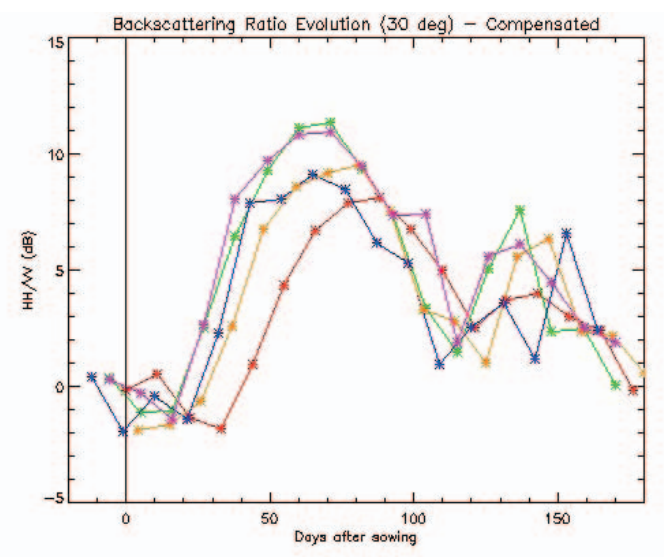

Fig. 2. Temporal evolution of the HH/VV ratio for all the monitored parcels at $30^{\circ}$ incidence

date. From the observation of Figs. 1 and 2, it is important to stress that the responses of all parcels but one (plotted in red) are very coincident, both for the individual $\sigma_{0}$ and for the HH/VV ratio. Therefore, we can conclude that these temporal signatures are associated with this rice crop type and, in principle, can be used for following its phenological evolution. The outlier parcel (in red) suffered a phenological delay due to water salinity, so this time series is also usefull to detect such cultivation anomalies.

The interpretation of the overall temporal evolution can be carried out by taking into account the phenological evolution of the plants. In general, the cultivation period is divided into 3 main phenological phases: vegetative, reproductive and mature [1]. During the vegetative stage the plant increases its height from 0 to about $70-90 \mathrm{~cm}$. It is composed of up to 10 stems (the main one and new ones appearing during this period), and some leaves at the top in the late part of this phase. Then, the appearance of the panicle defines the transition to the reproductive stage. In the reproductive stage there also appears a cluster of curved leaves in the top of the plants, as well as flowering elements. Moreover, the stems become drier and tend to present a cane structure. Finally, in the mature phase the grains appear and develop. The water content of the grains changes up and down until it is about 18-22 percent before harvesting. The vegetative phase finished about day 65-70 after sowing (mid August), and the mature phase started around 45 days later (end of September). Note that the maximum value of the $\mathrm{HH} / \mathrm{VV}$ ratio is coincident with the transition between vegetative and reproductive phase, whereas the decreasing trend after that maximum spans approximately the same time interval as the reproductive phase.

In addition to the power information, the co-polarimetric phase difference is also sensitive to the rice growing stages. This parameter (usually named as polarization phase difference, PPD, in the literature [2]), is computed as the phase of the complex cross correlation between two co-polarimetric

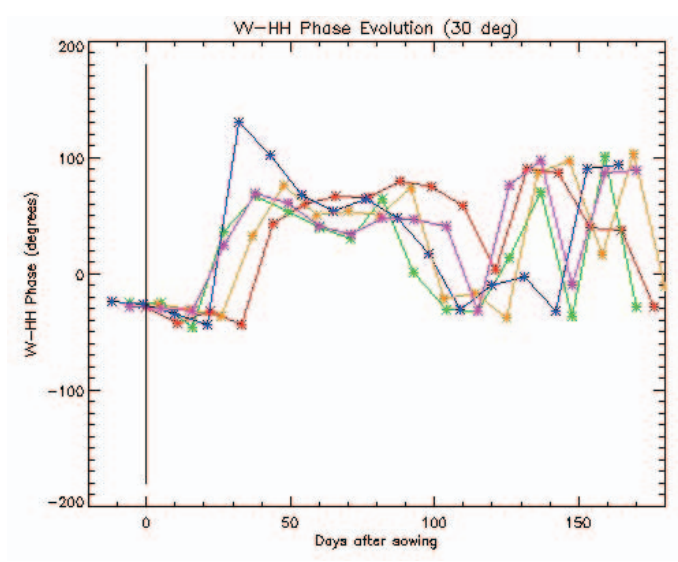

Fig. 3. Temporal evolution of the phase difference between $\mathrm{VV}$ and $\mathrm{HH}$ for all the monitored parcels at $30^{\circ}$ incidence
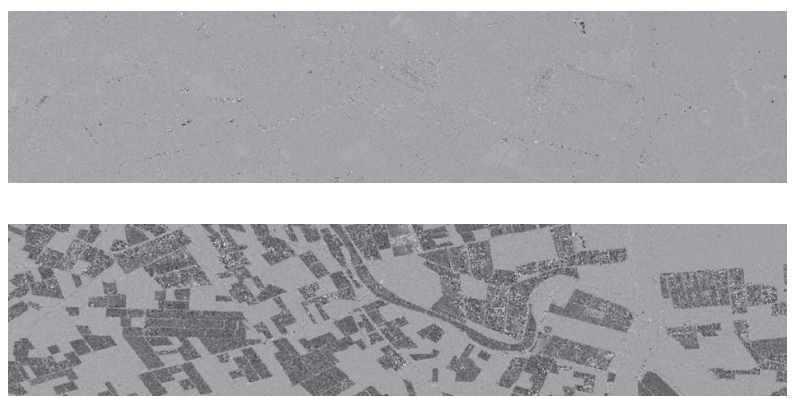

Fig. 4. Images of the PPD from a part of the test site. Dates: Top - May 30. Bottom - August 26

channels. In Fig. 3 a HHVV phase jump can be observed at 30 to 40 days after sowing. At this time the plants start to reach out of the water level and are visible. This could be used as an indicator for the plant emergence time. Further in time there are no significant phase variations observed, and the phase level keeps being the same until day 90-110, when the vegetative phase is ending.

Maps of the PPD for a part of the rice test site are shown in Fig. 4, corresponding to acquisition dates May 30 (about sowing date, i.e. low plant development stage) and August 26 (high plant development stage). A higher phase difference can be observed over the vegetated rice fields (dark color) that are significantly distinguishable from the non- or other crop type vegetated fields (lower image), whereas in the upper image no PPD is observed (gray color corresponding to zero PPD).

Exploiting the polarimetric information available, the dual-polarimetric decomposition especially designed for data with only two polarimetric channels, also named as $\mathrm{H} 2 \alpha$ [3], was investigated in terms of change detection occurring during the rice growing stages. The physical interpretation of the statistical polarimetric parameters, entropy $(H)$ and alpha $(\alpha)$, is based on two orthogonal scattering mechanisms and not on three as described for the original eigenvector decom- 

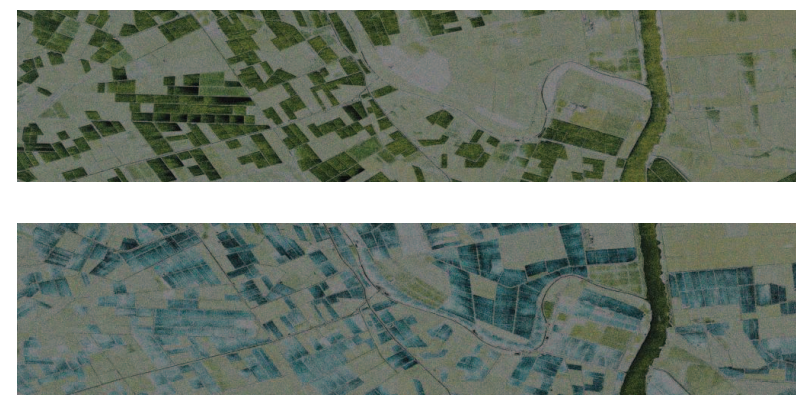

Fig. 5. HSV false color composite images from a part of the test site, obtained with the $\mathrm{H} 2 \alpha$ decomposition (H: alpha angle, S: entropy, V: total power). Dates: Top - May 30. Bottom - August 26

position in [4]. Accordingly, low entropy characterizes one dominant scattering mechanism occurring within one resolution cell, and for high entropy more than one scattering mechanism is involved. The interpretation for the alpha parameter is more difficult as a part of the vegetation volume component is mixed with the dihedral component and only the surface component, when vegetation is very low, can be distinguished. In Fig. 5, false color composite images are presented for the same part of the test site and the same dates as Fig. 4. A significant difference between the two dates can be observed, since the vegetation structure generates a polarimetric signature not present at the beginning of the season. Moreover, cultivated and not cultivated fields can be distinguished in the image on August 26 by a simple inspection.

More significant changes can be observed in the 2D plot representation on the entropy-alpha plane seen in Fig. 6. On May 30 the highest intensity (dark red) of the plot is located in the low to medium entropy and low alpha angle region. This corresponds to a non to very sparse vegetated area. Whereas for August 26 two centers of point clouds and a slight increase of the previous existing cloud center are observed. The increase of the former cloud is interpreted with the increase of existing vegetation on all fields in the scene. In addition a very distinct second cloud with medium high entropy and medium high alpha angle can be separated. The second cloud of points is generated by the cultivated fields because of their scattering behavior ( $\mathrm{HH}$ much stronger than VV due to differential extinction and double bounce dominance) which is very particular of such fields.

\section{INSAR RESULTS}

In order to test the potential application of X-band repeat-pass satellite interferometry for agriculture monitoring we have also computed interferograms by using pairs of successive images. Although the 11-days revisit cycle of TerraSAR-X is short when compared to the rest of remote sensing satellites, it still appears to be too large for agriculture applications

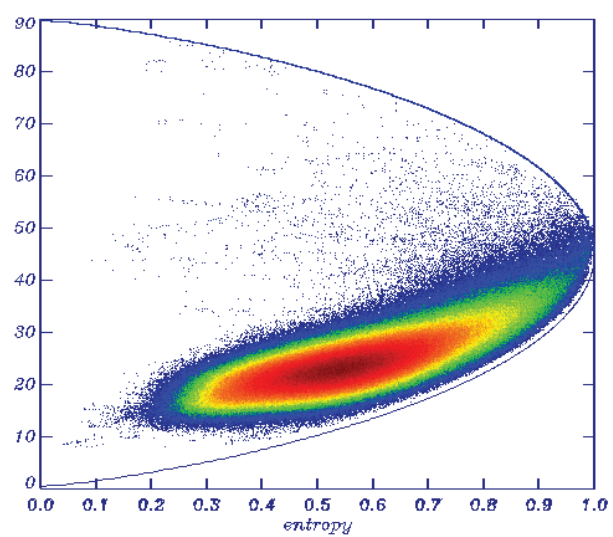

(a)

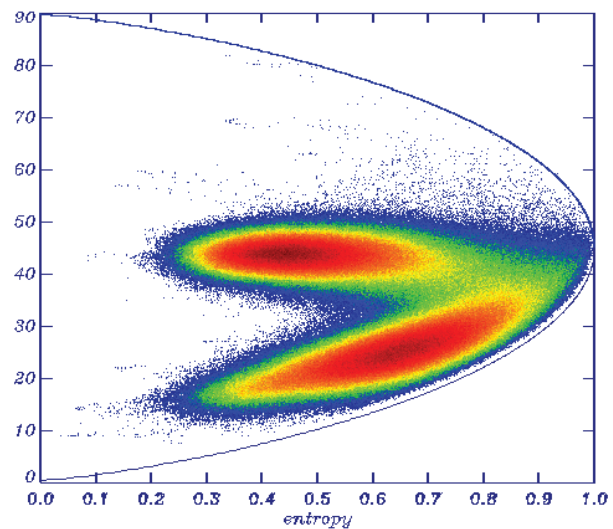

(b)

Fig. 6. Representation of the data from a part of the test site in the entropy-alpha plane. (a) May 30, (b) August 26

because vegetation develops very fast in crops. Fig. 7 shows the evolution of the interferometric coherence obtained by the $\mathrm{HH}$ images over a bare field and a rice field (parcel \#34). Coherence keeps a high level (above 0.8) on the bare fields for most of the interferograms, being the exceptions due to severe rain and wind events. Instead, coherence is quite low on the rice field for many interferograms as a consequence of the temporal decorrelation caused by the vegetation evolution itself. Nonetheless, it is above 0.5 for one interferogram, and above 0.4 for other two.

The interferogram with best coherence on the rice parcel \#34 is shown for the whole test site in Fig. 8. Despite the low coherence over the cultivated fields (clearly identified in the coherence map as dark areas) in contrast to the bare fields (bright areas), the phase still preserves some information content. An interesting feature is the variation of the interferometric phase between adjacent rice fields, which can be observed in many areas. For instance, there is a region of rectangular fields at the east of the river (center-right part of the images) where we can distinguish a mosaic of fields, 


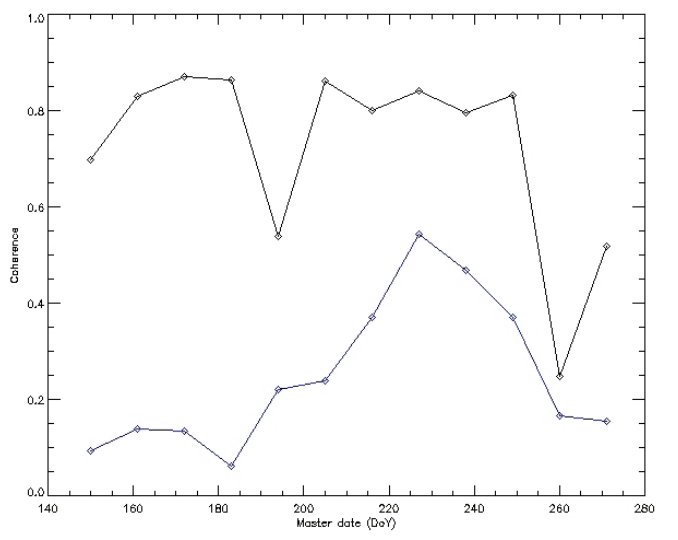

Fig. 7. Temporal evolution of the interferometric coherence for the $\mathrm{HH}$ channel at parcel \#34 (blue) and a nearby bare field (black). Interferograms are computed with pairs of images separated 11 days.

each one with an homogeneous phase, but different among them. Note that the high resolution of the images and the large size of the fields (more than 10 ha in all cases) allows enough multilooking for ensuring good estimations of coherence and phase at parcel scale. Further investigation about the actual phase values and the possible retrieval of vegetation height is still needed.

\section{CONCLUSIONS}

The HH/VV, the phase difference between HH and VV channels, and the dual-polarimetric entropy-alpha decomposition have demonstrated some potential in rice monitoring, rice mapping and detection of particular phenological stages. In addition, some preliminary results with interferometry have been obtained, which are subject to further interpretation.

\section{ACKNOWLEDGMENT}

All SAR images have been provided by DLR in the framework of projects LAN0021 and LAN0234 of the pre-launch AO of TerraSAR-X. The authors would like to thank the support of M. Cano, F. Carrascal and S. Aparicio, all from the Federacion de Arroceros de Sevilla, for providing the ground measurement data.

\section{REFERENCES}

[1] U. Meier, Ed., Growth Stages of Mono- and Dicotyledonous Plants. BBCH Monograph, 2nd edition, 2001, http://www.bba.de/veroeff/bbch/bbcheng.pdf.

[2] F. T. Ulaby, D. N. Held, M. C. Dobson, K. C. McDonald, and T. B. A. Senior, "Relating polarization phase differ-

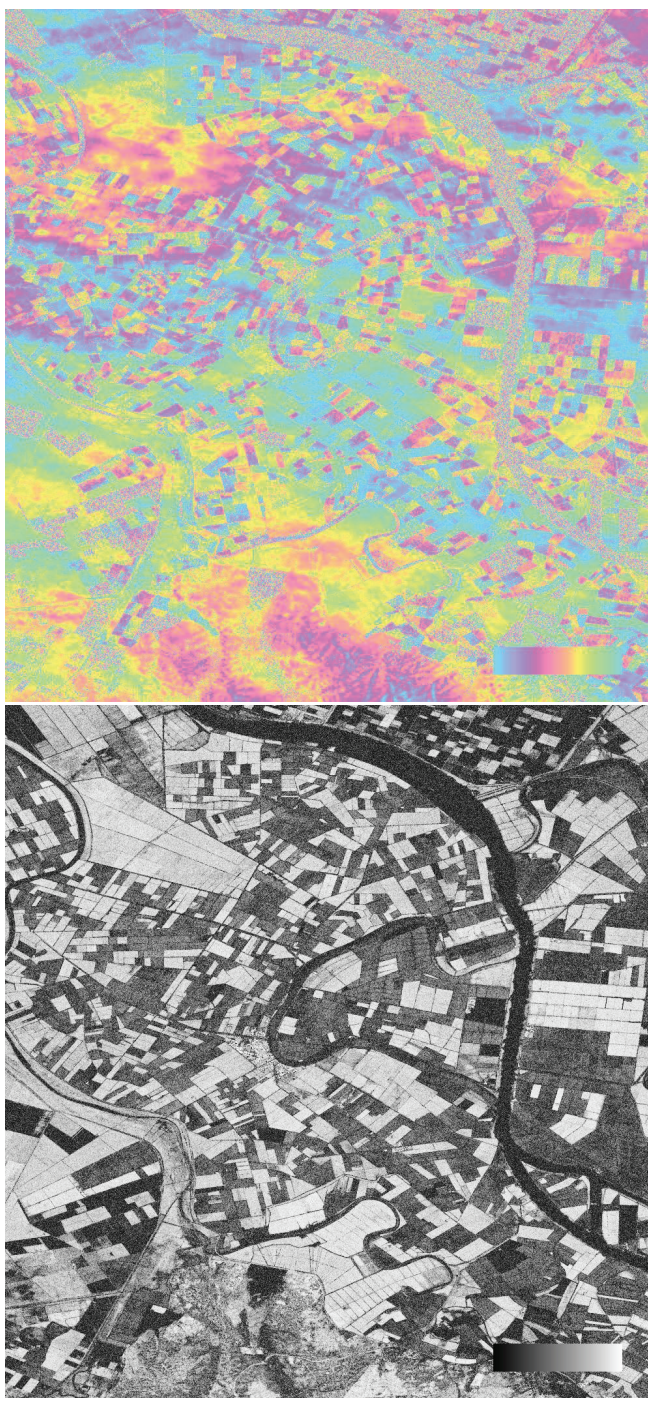

Fig. 8. HH interferogram formed by images on August 15 and August 26 (DoY 227 and 238) over the whole test site. Top: phase. Bottom: coherence

ence of SAR signals to scene properties," IEEE Trans. Geosci. Remote Sensing, vol. 25, no. 1, pp. 83-92, Jan. 1987.

[3] S. R. Cloude, "The dual polarization entropy/alpha decomposition: a PALSAR case study," in Proceedings of the 3rd PolInSAR Workshop, Frascati, Italy, Jan. 2007.

[4] S. R. Cloude and E. Pottier, "An entropy based classification scheme for land applications of polarimetric SAR," IEEE Trans. Geosci. Remote Sensing, vol. 35, no. 1, pp. 68-78, Jan. 1997. 